\title{
Opinión
}

\section{Medicina basada en evidencia y políticas de salud pública, el caso de la nueva Ley de Tránsito}

\author{
(Evidence-Based Medicine and Public Health Policies, \\ the case of the new Traffic Law)
}

\author{
Ricardo Millán-González
}

\section{Resumen:}

La medicina basada en evidencia es una corriente metodológica que permite discriminar la información existente a nivel mundial, con la intención de aclarar dudas clínicas y así tomar las mejores decisiones para una población determinada. Un ámbito donde existe un adecuado sustento científico es aquel que vincula el consumo de licor y la conducción de vehículos con una serie de variables epidemiológicas, con un importante aumento del riesgo de accidentes, y con el hecho de que medidas preventivas son capaces de reducir la mortalidad por esta causa. Por lo tanto, se cuestionan los niveles tolerados por la actual Ley de Tránsito y la ausencia de mayores restricciones a los grupos etáreos más propensos a accidentarse cuando se ha ingerido alcohol. Es indispensable la aplicación de estos conceptos en la legislación nacional, y en ello el Colegio de Médicos y Cirujanos de Costa Rica tiene un papel preponderante.

Descriptores: medicina basada en evidencia, Ley de Tránsito, consumo de licor y conducción de vehículos

\begin{abstract}
:
Evidence Based Medicine is a methodological approach that allows us to discriminate worldwide available information with the intention to clarify clinical questions and thus make the best decisions for a given population. One area where there is adequate scientific support is the one that links the consumption of alcohol and driving with a series of epidemiological variables, with a significant increased risk of accidents and with the fact that preventive measures are able to reduce the mortality rate. Therefore the current Traffic Law is deeply questioned in two areas: the allowed levels of blood concentration of alcohol and the absence of
\end{abstract}

Médico psiquiatra, especialista en Psiquiatría de Enlace, profesor de psiquiatría de grado y postgrado, UCR, Hospital Nacional de Geriatría y Gerontología

Correo electrónico: ricardo.millangonzalez@ucr.ac.cr

ISSN 0001-6002/2011/53/3/149-150

Acta Médica Costarricense, (2011

Colegio de Médicos y Cirujanos restrictions on the age groups most prone to accidents when alcohol is ingested. It is necessary to apply these concepts in the national legislation, and for that the College of Physicians and Surgeons of Costa Rica has a preponderant role.

Key-words: Evidence-based Medicine, Traffic Law, alcohol consumption and driving of vehicles.

Recibido: 31 de enero de 2011 Aceptado: 28 de abril de 2011

La medicina basada en evidencia ${ }^{1}$ se refiere a la revolucionaria corriente metodológica propuesta en la década de los noventa por el profesor Gordon Guyatt, de la Universidad de MacMaster, en Ontario, Canadá. Consiste en seguir una secuencia de recolección, clasificación y evaluación de los datos científicos existente a nivel mundial, con la intención de contestar una duda clínica inicial, y de esta forma elegir la opción más adecuada y balanceada para el beneficio de una población específica de pacientes. $\mathrm{Su}$ auge ha sido tal, que es considerada el estándar de oro para la toma de decisiones clínicas y científicas.

En poco tiempo, esta propuesta se diseminó y hoy se emplea en cualquier área de la medicina. De esta manera, se desplazaron las dinámicas históricamente más relevantes, como "hacer las cosas por experiencia personal" (lo que se sabe es proclive a los sesgos individuales de apreciación) o siguiendo un razonamiento fisiopatológico (revertir un proceso mediante intervenciones contrarias a dicha condición, lo cual, a pesar de una lógica inicial, no siempre permite alcanzar los resultados esperados).

A pesar de la creciente investigación en todos los campos de la medicina, frecuentemente es difícil encontrar una sólida evidencia que conteste una pregunta determinada, ya sea por ausencia de estudios que aborden un tema específico, o por aplicación de una metodología inadecuada $y$, por lo tanto, con conclusiones sesgadas. Sin embargo, a nivel de salud pública, un ámbito donde existe un fuerte sustento científico es aquel que vincula el consumo de licor y la conducción de vehículos, con una serie de variables 
epidemiológicas, con un importante aumento del riesgo de accidentes, y con el hecho de que medidas preventivas (represivas y educativas, habiendo demostrado las segundas ser más efectivas que las primeras) son capaces de reducir la mortalidad por esta causa.

Así, a la luz de la reciente polémica producto de la nueva Ley de Tránsito, ${ }^{2}$ sobre todo en lo relacionado con la conducción bajo los efectos del licor, cabe formular las siguientes preguntas: ¿Existe algún grupo etario particularmente proclive a los accidentes de tránsito cuando se conduce bajo los efectos del alcohol? ¿Se ha descrito una asociación entre la concentración plasmática de alcohol en sangre y el riesgo de accidentes? ¿Se ha logrado descender estos riesgos con la aplicación de medidas restrictivas?

En una reciente evaluación sistemática de la bibliografía de este tema realizada por el Dr. Álvaro Ruiz y colaboradores, ${ }^{3}$ del Departamento de Epidemiología y Bioestadística de la Pontificia Universidad Javeriana de Bogotá, Colombia, se contestan esas preguntas. A saber:

Uno de los principales factores de riesgo cuando se maneja y se ha ingerido previamente licor, es la edad del conductor. En menores de 30 años (asumiendo que la conducción inicia con la mayoría de edad), se incrementa de forma desproporcionada la posibilidad de un accidente, y de que en este ocurra una fatalidad.

Lo anterior se explica por diferentes razones: una menor experiencia en la conducción y una mayor tendencia a adoptar conductas de riesgo e incapacidad para moderar el consumo de licor en ausencia de adicción. Desde el punto de vista neurobiológico, lo anterior estaría determinado por inmadurez neuronal y de conexiones en la corteza prefrontal, la cual confiere funciones cerebrales como la atención y la memoria de trabajo, así como la capacidad de inhibir conductas desadaptativas o impulsivas. ${ }^{3-4}$

En la revisión del Dr. Ruiz se aprecia que niveles de alcohol en sangre superiores al $0 \%$, incrementan el riesgo de accidentes de tránsito, y que este asciende de forma exponencial conforme lo hace la concentración sanguínea. De esta manera, la accidentalidad se incrementa de 6 a 17 veces con concentraciones plasmáticas de $0,05 \mathrm{mg} / \mathrm{dl}$, de 11 a 52 veces en rangos superiores de $0,08 \mathrm{mg} / \mathrm{dl}$, y alcanza un máximo de 244 veces por encima de $0,15 \mathrm{mg} / \mathrm{dl}$.

Pero quizás lo más relevante de esta información es que en países donde se han instaurado medidas restrictivas de conducción bajo los efectos del alcohol, conforme se desciende en el rango aceptado, se reducen accidentes y mortalidad. ${ }^{3}$ Así, cuando en Canadá se impusieron restricciones por encima de $0,08 \mathrm{mg} / \mathrm{dl}$, la mortalidad general en carreteras decreció un 6\%; cuando el rango permitido en Holanda fue de $0,05 \mathrm{mg} / \mathrm{dl}$, hubo un descenso del 15\% de los accidentes de tránsito relacionados con alcohol, y en Suecia, a partir de la reducción del límite sancionado de 0,05 a 0,02 $\mathrm{mg} / \mathrm{dl}$ (lo que se conoce como tolerancia cero), se reportó un descenso de casi el $10 \%$ de los accidentes mortales. Experiencias similares se describen en los Estados Unidos, Francia, Austria, Australia y otros países. ${ }^{3}$

La información científica pone en entredicho que el límite permitido en la Ley de Tránsito vigente ${ }^{2}$ sea de 0,05 $\mathrm{mg} / \mathrm{dl}$; que exista un comodín adicional, conocido como preebriedad, que tolera niveles entre ese rango y $0,075 \mathrm{mg} / \mathrm{dl}, \mathrm{y}$ que no se hayan formulado mayores restricciones en los grupos de edades más proclives a accidentarse. Con la evidencia existente es indiscutible que en menores de 30 años, no debiera tolerarse rangos distintos al $0 \%$ de alcohol en sangre.

La aplicación de la ciencia al servicio de las necesidades de la población es una práctica indispensable en la legislación nacional. Por lo tanto, la participación del Colegio de Médicos y Cirujanos de Costa Rica (en particular de los especialistas en las áreas de psiquiatría, adictología, toxicología, traumatología, emergenciología y salud pública) en la discusión sobre cualquier enmienda a la actual ley, o en la eventual formulación de una nueva, debe ser preponderante, firme, enérgica y protagónica.

Se superaría así la tendencia de tomar decisiones sustentadas en puntos de vista personales, juicios de valor, presiones políticas e interpretaciones culturales del entorno, y se evolucionaría entonces hacia la utilización de la medicina basada en evidencia al servicio de la salud pública y del bienestar de los costarricenses.

\section{Referencias}

1. Guyatt G, Rennie D, Meade MO, Cook DJ. JAMA evidence: Users' guidelines to the medical literature. Second Ed. New York, New York: McGraw-Hill Medical; 2008.

2. Ley de Tránsito por Vías Públicas Terrestres: Número 7331 del 13 de abril de 1993 y sus reformas. Sistema Nacional de Legislación Vigente (SINALEVI). Accedido 13 de diciembre de 2010. En: http://www. transito.go.cr/legislacion/leydetransito.html.

3. Ruiz A, Macías F, Gómez-Restrepo C, Rondón M, Lozano JM. Niveles de alcohol en sangre y riesgo de accidentalidad vial: revisión sistemática de la literatura. Revista Colombiana de Psiquiatría 2010, 39 (supl): 249-278.

4. Stahl SM. Circuits in Psychopharmacology. In: Stahl's Essential Psychopharmacology: Neuroscientific Basis and Practical Applications. Third Edition: Cambridge University Press, 2008: 195222. 